DOI: 10.21915 /BIMAS.2019105

\title{
71 HOLOMORPHIC VERTEX OPERATOR ALGEBRAS OF CENTRAL CHARGE 24
}

\author{
CHING HUNG LAM $^{1, a}$ AND HIROKI SHIMAKURA ${ }^{2, b}$ \\ Dedicated to Robert L. Griess, Jr on the occasion of his $71^{\text {st }}$ birthday \\ ${ }^{1}$ Institute of Mathematics, Academia Sinica, Taipei 10617, Taiwan. \\ ${ }^{a}$ E-mail: chlam@math.sinica.edu.tw \\ ${ }^{2}$ Graduate School of Information Sciences, Tohoku University, Sendai 980-8579, Japan. \\ ${ }^{b}$ E-mail: shimakura@tohoku.ac.jp
}

\begin{abstract}
In this article, we give a survey on the recent progress towards the classification of strongly regular holomorphic vertex operator algebras of central charge 24. In particular, we review the construction of the holomorphic vertex operator algebras that realize the 71 Lie algebras in Schellekens' list. In addition, we discuss an open question if the Lie algebra structure of the weight one subspace will determine the isomorphism class of a holomorphic vertex operator algebra of central charge 24 uniquely.
\end{abstract}

\section{Introduction}

The classification of strongly regular holomorphic vertex operator algebras (VOAs) of central charge 24 is one of the fundamental problems in vertex operator algebras and conformal field theory. Here, strongly regular is defined to be rational, $C_{2}$-cofinite, self-contragredient, and of CFT-type.

Received March 19, 2017 and in revised form March 11, 2018.

AMS Subject Classification: Primary 17B69.

Key words and phrases: Holomorphic vertex operator algebra, affine lie algebra, orbifold construction.

C. H. Lam was partially supported by MoST grant 104-2115-M-001-004-MY3 of Taiwan

H. Shimakura was partially supported by JSPS KAKENHI Grant Numbers 26800001 and $17 \mathrm{~K} 05154$.

C. H. Lam and H. Shimakura were partially supported by JSPS Program for Advancing Strategic International Networks to Accelerate the Circulation of Talented Researchers "Development of Concentrated Mathematical Center Linking to Wisdom of the Next Generation". 
In general, the weight one subspace of a VOA of CFT-type has a Lie algebra structure via the 0-th mode [2], which we often call the weight one Lie algebra. Let $V$ be a strongly regular holomorphic VOA of central charge 24 . Then the weight one Lie algebra $V_{1}$ is zero, abelian of rank 24 or semisimple ([15]); if $V_{1}$ is abelian of rank 24, then $V$ is isomorphic to the Leech lattice $\operatorname{VOA}([16])$; if $V_{1}$ is zero, then it is conjecturally isomorphic to the moonshine $\operatorname{VOA}([25])$.

We now assume that $V_{1}$ is semisimple. Then for each simple ideal of $V_{1}$, there is an equation that relates the dual Coxeter number, the level of its affinization and the dimension of $V_{1}([53,15])$. It turns out that there are exactly 221 solutions for these equations. Here, the level must be a positive integer since $V$ is $C_{2}$-cofinite $([15])$. Schellekens [53] (see also [22]) found additional constraints and gave a list of possible 69 semisimple Lie algebra structures for $V_{1}$, which is called Schellekens' list. Let $U$ be the subVOA generated by $V_{1}$. Then $U$ is the tensor product of simple affine VOAs at positive integral levels $([17])$ and it is full ([15]), i.e., its conformal vector coincides with that of $V$. Hence $V$ is a direct sum of $U$ and finitely many irreducible $U$-submodules with integral conformal weight at least 2. By the classification of irreducible modules for simple affine VOAs ([26]), it seems that there are a few possibilities of $U$-module structures of $V$ and the VOA structure of $V$ would be determined uniquely by the structure of the weight one Lie algebra.

For us, such a situation may also be viewed as a VOA analogue of the classification of (positive-definite) even unimodular lattices of rank 24 . It is a famous theorem by Niemeier [51] (cf. [55]) that there are exactly 24 even unimodular lattices of rank 24 and their isometry types are determined by their root systems, a combinatorial structure of norm 2 vectors. It seems that the weight one Lie algebra of a holomorphic VOA of central charge 24 play a similar role as the root system of the even unimodular lattices of rank 24. In particular, there exists a unique even unimodular lattice of rank 24 which has no vectors of norm 2 and it is isometric to the Leech lattice [5]. Hence the characterization of the moonshine VOA mentioned above would be a VOA analogue of that of the Leech lattice. 
When Schellekens [53] obtained his list, not all 71 cases were known explicitly at that time. Besides, it is still an open question if the Lie algebra structure of the weight one subspace will determine the VOA structure uniquely when the central charge is 24 . In the recent years, there is much progress towards the classification. Schellekens' list has been verified mathematically by van Ekeren, Möller and Scheithauer [22, 49]. Moreover, holomorphic VOAs associated with all 71 Lie algebras in the list have been constructed. We list the constructions in chronological order as follows:

- 24 Niemeier lattice VOAs $V_{N}[2,25,8]$;

- 15 obtained by $\mathbb{Z}_{2}$-orbifold constructions associated with $V_{N}$ and the -1 -isometry $[25,7]$;

- 17 framed holomorphic VOAs [31, 33];

- 3 obtained by $\mathbb{Z}_{3}$-orbifold constructions associated with $V_{N}$ and order 3 isometries $[46,52]$;

- 5 obtained by $\mathbb{Z}_{n}$-orbifold constructions associated with $V_{N}$ and some isometries of order $n \geq 4[22,49]$;

- 5 obtained by $\mathbb{Z}_{2}$-orbifold constructions associated with inner automorphisms [35];

- 1 obtained by $\mathbb{Z}_{7}$-orbifold construction associated with the Leech lattice VOA; the corresponding order 7 automorphism is the product of an inner automorphism and a standard lift of order 7 isometry of the Leech lattice [36];

- 1 obtained by Mirror extension and $\mathbb{Z}_{2}$-orbifold construction [32].

Therefore, the following theorem was established:

Theorem 1.1. Let $\mathfrak{g}$ be one of the 71 Lie algebras in Schellekens' list. Then there exists a strongly regular holomorphic VOA of central charge 24 whose weight one Lie algebra is isomorphic to $\mathfrak{g}$. 
The uniqueness of several holomorphic VOAs of central charge 24 have also been established [16, 34, 30, 37] (see also Remark 6.10 for the results after submission of this article).

In this article, we will give a survey on the recent progress towards the classification of strongly regular holomorphic VOAs of central charge 24 . In particular, we will review constructions of the holomorphic VOAs that realize the Lie algebras in Schellekens' list. Several main techniques will also be discussed. In addition, we will discuss the uniqueness conjecture and review some of the known results.

The organization of the article is as follows. In Section 2, we review some basic notions about VOAs and their weight one Lie algebras. In Section 3, we describe a strategy for the classification of holomorphic VOAs of central charge 24 based on Schellekens' list [53]. In Section 4, we review several main techniques for the construction of holomorphic VOAs of central charge 24 , which include the $\mathbb{Z}_{n}$-orbifold construction [22, 49], Li's $\Delta$-operator [43] and certain dimension formulas on the weight one Lie algebras [50, 49]. In Section 5, we review the constructions of 71 holomorphic VOAs that realize the Lie algebras in Schellekens' list. We also discuss how to use the techniques in Section 4 for the constructions. In Section 6, we review some known results about the uniqueness problem and discuss a technique which we call "reverse orbifold construction" [37]. We also discuss its application towards the uniqueness problem.

\section{Preliminary}

Throughout this article, all VOAs are defined over the field $\mathbb{C}$ of complex numbers. We recall the notion of vertex operator algebras (VOAs) and (twisted) modules from $[2,25,24,13]$.

A vertex operator algebra $(\mathrm{VOA})(V, Y, \mathbb{1}, \omega)$ is a $\mathbb{Z}$-graded vector space $V=\bigoplus_{m \in \mathbb{Z}} V_{m}$ equipped with a linear map

$$
Y(a, z)=\sum_{i \in \mathbb{Z}} a_{(i)} z^{-i-1} \in(\operatorname{End}(V))\left[\left[z, z^{-1}\right]\right], \quad a \in V,
$$

the vacuum vector $\mathbb{1}$ and the conformal vector $\omega$ satisfying a number of conditions $([2,25])$. We often denote it by $V$. For $a \in V$ and $n \in \mathbb{Z}$, we 
often call $a_{(n)}$ the $n$-th mode of $a$. Note that $L(n)=\omega_{(n+1)}$ satisfy the Virasoro relation:

$$
\left[L_{(m)}, L_{(n)}\right]=(m-n) L_{(m+n)}+\frac{1}{12}\left(m^{3}-m\right) \delta_{m+n, 0} c \mathrm{id}_{V},
$$

where $c$ is a complex number, called the central charge of $V$.

A linear automorphism $g$ of $V$ is called a (VOA) automorphism of $V$ if

$$
g \omega=\omega \quad \text { and } \quad g Y(v, z)=Y(g v, z) g \quad \text { for all } v \in V .
$$

A vertex operator subalgebra (or a subVOA) is a graded subspace of $V$ which has a structure of a VOA such that the operations and its grading agree with the restriction of those of $V$ and that they share the vacuum vector. When they also share the conformal vector, we will call it a full subVOA. For an automorphism $g$ of a VOA $V$, let $V^{g}$ denote the set of fixed-points of $g$. Note that $V^{g}$ is a full subVOA of $V$.

A VOA is said to be rational if its admissible module category is semisimple, and a rational VOA is said to be holomorphic if it itself is the only irreducible module up to isomorphism. A VOA is said to be of CFT-type if $V_{0}=\mathbb{C} \mathbb{1}$ (note that $V_{n}=0$ for all $n<0$ if $V_{0}=\mathbb{C} \mathbb{1}[18$, Lemma 5.2]), and is said to be $C_{2}$-cofinite if the codimension in $V$ of the subspace spanned by the vectors of form $u_{(-2)} v, u, v \in V$, is finite. A module is said to be selfcontragredient if its contragredient module is isomorphic to itself. A VOA is said to be strongly regular if it is rational, $C_{2}$-cofinite, self-contragredient and of CFT-type. Note that a strongly regular VOA is simple.

Let $g$ be an automorphism of a VOA $V$ of order $n \in \mathbb{Z}_{>0}$ and let $M$ be an irreducible $g$-twisted $V$-module. Note that if $g=1$, then $M$ is just an irreducible $V$-module. Then the twisted module $M$ can be decomposed as $M=\bigoplus_{i \in(1 / n) \mathbb{Z}_{\geq 0}} M_{i+w}$, where $w \in \mathbb{C}, M_{w} \neq 0$ and $M_{s}=\{v \in M \mid L(0) v=$ $s v\}$. The number $w$ is called the conformal weight of $M$. For the definition of (twisted) modules, see [24, 13].

Let $V$ be a VOA of CFT-type. Then, the weight one space $V_{1}$ has a Lie algebra structure via the 0 -th mode $([2,25])$, which we often call the weight one Lie algebra of $V$. Moreover, the $n$-th modes $v_{(n)}, v \in V_{1}, n \in \mathbb{Z}$, define an affine representation of the Lie algebra $V_{1}$ on $V$. For a simple Lie subalgebra $\mathfrak{a}$ of $V_{1}$, the level of $\mathfrak{a}$ is defined to be the scalar by which the 
canonical central element acts on $V$ as the affine representation. When the type of the root system of $\mathfrak{a}$ is $X_{n}$ and the level of $\mathfrak{a}$ is $k$, we denote the type of $\mathfrak{a}$ by $X_{n, k}$. The following proposition allows us to study a strongly regular VOA via the representation theory of simple affine VOAs at positive integral level. For the explicit construction of affine VOAs, we will refer to [26] for details.

Proposition 2.1 ([17, Theorem 1.1, Corollary 4.3]). Let $V$ be a strongly regular VOA. Then $V_{1}$ is reductive. Let $\mathfrak{s}$ be a simple Lie subalgebra of $V_{1}$. Then $V$ is an integrable module for the affine representation of $\mathfrak{s}$ on $V$, and the subVOA generated by $\mathfrak{s}$ is isomorphic to the simple affine VOA associated with $\mathfrak{s}$ at positive integral level.

Now, we assume that $V$ is strongly regular and $V_{1}$ is semisimple. We also assume that the subVOA $L\left(V_{1}\right)$ generated $V_{1}$ is full. Then by the proposition above, $L\left(V_{1}\right)$ is the tensor product of simple affine VOAs and hence it is rational. Since $L\left(V_{1}\right)$ is full, $V$ is the direct sum of finitely many irreducible $L\left(V_{1}\right)$-modules. In fact, if the weight one Lie algebra of a (strongly regular) holomorphic VOA of central charge 24 is neither zero nor abelian of rank 24, then the VOA satisfies these assumptions by the following proposition:

Proposition 2.2 ([15, (1.1), Theorem 3 and Proposition 4.1]). Let $V$ be a strongly regular holomorphic VOA of $c=24$. If the Lie algebra $V_{1}$ is neither $\{0\}$ nor abelian of rank 24 , then $V_{1}$ is semisimple, and the subVOA generated by $V_{1}$ is full, i.e., its conformal vector coincides with that of $V$. In addition, for any simple ideal of $V_{1}$ at level $k$, the identity

$$
\frac{h^{\vee}}{k}=\frac{\operatorname{dim} V_{1}-24}{24}
$$

holds, where $h^{\vee}$ is the dual Coxeter number of the simple ideal.

This proposition restricts the structure of the semisimple weight one Lie algebra of a holomorphic VOA of central charge 24; indeed, there are only 221 possibilities for them $([53,22])$. 


\section{Towards the Classification of Holomorphic VOAs of Central Charge 24}

In this section, we describe a strategy for the classification of holomorphic VOAs of $c=24$. Let $V$ be a (strongly regular) holomorphic VOA. By the modular invariance on the space generated by the character of irreducible modules ([57]), the character of $V$ belongs to $\mathbb{C}\left[j^{1 / 3}\right]$ (cf. [27]), where $j$ is the famous $j$-function. This implies that the central charge $c$ of $V$ must be a multiple of 8 . In [16, Theorems 1 and 2], it was proved that any holomorphic VOA of $c=8$ (resp. $c=16$ ) is isomorphic to the lattice VOA $V_{E_{8}}$ (resp. the lattice VOAs $V_{E_{8} \oplus E_{8}}$ or $\left.V_{D_{16}^{+}}\right)$. For the case $c=24$, Schellekens gave a list of possible 71 weight one Lie algebra structures of holomorphic VOAs of $c=24([53])$; see Appendix for the list. Note that in $[22,49]$, this list was reproved mathematically. By Schellekens' list, the classification problem of $c=24$ can be divided into the following two steps:

(1) For every Lie algebra $\mathfrak{g}$ in the list, construct a (strongly regular) holomorphic VOA of $c=24$ whose weight one Lie algebra is isomorphic to $\mathfrak{g}$.

(2) Show that the isomorphism class of a holomorphic VOA of $c=24$ is uniquely determined by the Lie algebra structure of the weight one space.

Step (1) has been completed recently; see Sections 4 and 5 for detail. Step (2) is in progress; see Section 6 for the current situation.

Remark 3.1. When $c \geq 32$, it seems that the classification of holomorphic VOAs is impossible; for example, there are at least $8 \times 10^{7}$ (non-isometric) positive-definite even unimodular lattices of rank 32 (cf. [6]), and hence there are at least $8 \times 10^{7}$ (non-isomorphic) holomorphic lattice VOAs of $c=32$.

\section{Orbifold Construction, Li's $\Delta$-operator and Dimension Formulas}

In this section, we will review several main techniques for constructions of holomorphic VOAs of $c=24$. These techniques include the orbifold construction, Li's $\Delta$-operator and certain dimension formulas on the weight one Lie algebras.

\section{1. $\mathbb{Z}_{n}$-orbifold construction}

In this subsection, we will review the $\mathbb{Z}_{n}$-orbifold construction associated 
with a holomorphic VOA and an automorphism of arbitrary finite order from $[22,49]$, which was inspired by Miyamoto's work [46] on the $\mathbb{Z}_{3}$-orbifold construction.

Let $V$ be a strongly regular holomorphic VOA. Let $g$ be a (finite) order $n$ automorphism of $V$. For $0 \leq i \leq n-1$, let $V\left[g^{i}\right]$ be the irreducible $g^{i}$ twisted $V$-module. Such a module exists and is unique by [13, Theorem 1.2]. Note that $V\left[g^{0}\right]=V$. Moreover, it is known that the group $\langle g\rangle$ acts on the twisted module $V\left[g^{j}\right]$ for each $1 \leq j \leq n-1$. More precisely, there exists $\phi_{j}:\langle g\rangle \rightarrow \operatorname{Aut}_{\mathbb{C}}\left(V\left[g^{j}\right]\right)$ such that for all $v \in V$ and $i \in \mathbb{Z}$,

$$
\phi_{j}\left(g^{i}\right) Y_{V\left[g^{j}\right]}(v, z) \phi_{j}\left(g^{i}\right)^{-1}=Y_{V\left[g^{j}\right]}\left(g^{i} v, z\right) .
$$

Note that such an action is unique up to a multiplication of an $n$-th root of unity. Set $\phi_{0}(g)=g \in \operatorname{Aut}(V)$. For $0 \leq j, k \leq n-1$, we denote

$$
W^{(j, k)}=\left\{w \in V\left[g^{j}\right] \mid \phi_{j}(g) w=e^{(2 \pi k \sqrt{-1}) / n} w\right\} .
$$

Let $V^{g}$ be the fixed-point subspace of $g$, which is a full subVOA of $V$. Note that $W^{(0,0)}=V^{g}$ and all $W^{(j, k)}$ 's are irreducible $V^{g}$-modules ([48, Theorem 2]). It was also shown recently by $[3,47]$ that $V^{g}$ is strongly regular. Moreover, any irreducible $V^{g}$-module is a submodule of $V\left[g^{i}\right]$ for some $i$, and there exist exactly $n^{2}$ non-isomorphic irreducible $V^{g}$-modules ([21]), which are represented by $\left\{W^{(j, k)} \mid 0 \leq j, k \leq n-1\right\}$. By calculating the $S$-matrix of $V^{g}([57,13])$, it was proved in $[22,49]$ that all irreducible $V^{g}$-modules $W^{(j, k)}$ are simple current modules. It implies that the set of isomorphism classes of irreducible $V^{g}$-modules, denoted by $R\left(V^{g}\right)$, forms an abelian group of order $n^{2}$ under the fusion product. We often identify an element in $R\left(V^{g}\right)$ with its representative irreducible $V^{g}$-module. Note that $R\left(V^{g}\right)=\left\{W^{(j, k)} \mid 0 \leq j, k \leq n-1\right\}$.

Remark 4.1. Explicit constructions of irreducible twisted modules have not been established yet for general automorphisms. However, the following cases are known:

- For a lattice VOA, irreducible twisted modules associated with a (standard) lift of an isometry of the lattice are constructed in $[41,11]$ (see Section 5.2).

- For a framed VOA, irreducible twisted modules associated with a product of $\tau$-involutions are constructed in [40] by using the fusion product. 
- For arbitrary VOAs, irreducible twisted modules associated with an inner automorphism are constructed by Li's $\Delta$-operator in [43] (see Section $4.2)$.

Now we assume the following:

(I) For $1 \leq i \leq n-1$, the conformal weight of $V\left[g^{i}\right]$ is positive.

(II) The conformal weight of $V[g]$ belongs to $(1 / n) \mathbb{Z}_{>0}$.

Then by $[22,49]$, the abelian group $R\left(V^{g}\right)$ is isomorphic to $\mathbb{Z}_{n} \times \mathbb{Z}_{n}$ and for any $M \in R\left(V^{g}\right)$, the conformal weight $q_{\Delta}(M)$ of $M$ belongs to $(1 / n) \mathbb{Z}$. Let $q: R\left(V^{g}\right) \rightarrow \mathbb{Z}_{n}$ be the map defined by

$$
q(M)=n \cdot q_{\Delta}(M) \quad(\bmod n)
$$

It was proved in $[22,49]$ that $q$ is a non-singular quadratic form on $R\left(V^{g}\right)$. In fact, the following theorem holds.

Theorem 4.2 ([22, 49]). Let $H$ be a totally isotropic subgroup of $R\left(V^{g}\right)$, i.e., $q(M)=0$ for all $M \in H$. Then, the $V^{g}$-module $\bigoplus_{M \in H} M$ has a strongly regular VOA structure as an $H$-graded simple current extension of $V^{g}$. Moreover, if $H$ is maximal, then $\bigoplus_{M \in H} M$ is holomorphic.

Under the assumptions (I) and (II), it is proved in [22] that one can choose the $\phi_{i}$ 's such that

- $W^{(i, j)} \bigotimes_{V^{g}} W^{(k, \ell)} \cong W^{(i+k, j+\ell)}$, where $\bigotimes_{V^{g}}$ is the fusion product of $V^{g}$ modules;

- $W^{(i, j)}$ has the conformal weight $q_{\Delta}\left(W^{(i, j)}\right)=j i / n \bmod \mathbb{Z}$.

Then $I=\left\{W^{(i, 0)} \mid 0 \leq i \leq n-1\right\}$ forms a maximal totally isotropic subgroup of $R\left(V^{g}\right)$ and by Theorem 4.2, the $V^{g}$-module

$$
\widetilde{V}_{g}=\bigoplus_{M \in I} M
$$

is a strongly regular holomorphic VOA. Since $I$ is isomorphic to $\mathbb{Z}_{n}, \widetilde{V}_{g}$ is a $\mathbb{Z}_{n}$-graded simple current extension of $V^{g}$. The construction of $\widetilde{V}_{g}$ is often called the $\mathbb{Z}_{n}$-orbifold construction associated with $V$ and $g$. Note that $W^{(1,0)}$ is the unique irreducible $V^{g}$-submodule of $V[g]$ with integral 
weights and that $I$ is the subgroup of $R\left(V^{g}\right)$ generated by $W^{(1,0)}$. Hence $\widetilde{V}_{g}$ is uniquely determined by $V$ and $g$, up to isomorphism.

\section{Remark 4.3.}

(1) Assume that $n$ is prime. Then for $1 \leq i \leq n-1$, the subspace of $V\left[g^{i}\right]$ with integral weights is an irreducible $V^{g}$-module. Such modules and $V^{g}$ form the maximal totally isotropic subgroup $I$ of $R\left(V^{g}\right)$.

(2) Let $g^{\prime}$ be an automorphism of $V$ which is conjugate to $g$. Then $g^{\prime}$ also satisfies the conditions (I) and (II), and $\widetilde{V}_{g^{\prime}}$ is isomorphic to $\widetilde{V}_{g}$ as a VOA.

\subsection{Li's $\Delta$-operator and associated twisted modules}

In this subsection, we review Li's $\Delta$-operator from [43], which gives an explicit construction of the irreducible twisted modules for inner automorphisms.

Let $V$ be a self-contragredient VOA of CFT-type. Let $\langle\cdot \mid \cdot\rangle$ be the invariant bilinear form on $V$ such that $\langle\mathbb{1} \mid \mathbb{1}\rangle=-1$ ([42]). Let $u \in V_{1}$ such that $u_{(0)}$ acts semisimply on $V$. Let $\sigma_{u}=\exp \left(-2 \pi \sqrt{-1} u_{(0)}\right)$ be the (inner) automorphism of $V$ associated with $u$. We assume that there exists a positive integer $n$ such that the spectrum of $u_{(0)}$ on $V$ belongs to $(1 / n) \mathbb{Z}$. Then we have $\sigma_{u}^{n}=1$ on $V$. Conversely, if $\sigma_{u}^{n}=1$, then the spectrum of $u_{(0)}$ on $V$ belongs to $(1 / n) \mathbb{Z}$. Let $\Delta(u, z)$ be Li's $\Delta$-operator defined in [43], i.e.,

$$
\Delta(u, z)=z^{u_{(0)}} \exp \left(\sum_{i=1}^{\infty} \frac{u_{(i)}}{-i}(-z)^{-i}\right) .
$$

Proposition 4.4 ([43, Proposition 5.4]). Let $\sigma$ be a finite order automorphism of $V$ and let $u \in V_{1}$ be as above such that $\sigma(u)=u$. Let $\left(M, Y_{M}\right)$ be a $\sigma$-twisted $V$-module and define $\left(M^{(u)}, Y_{M^{(u)}}(\cdot, z)\right)$ as follows:

$$
\begin{aligned}
& M^{(u)}=M \quad \text { as a vector space; } \\
& Y_{M^{(u)}}(a, z)=Y_{M}(\Delta(u, z) a, z) \quad \text { for any } a \in V .
\end{aligned}
$$

Then $\left(M^{(u)}, Y_{M^{(u)}}(\cdot, z)\right)$ is a $\sigma_{u} \sigma$-twisted $V$-module. Furthermore, if $M$ is irreducible, then so is $M^{(u)}$.

Later, we will discuss the orbifold construction associated with inner automorphisms. For this purpose, we will discuss the conditions (I) and (II) 
in the previous subsection. By the definition of the $\Delta$-operator, the weight operator acts on $V^{(u)}$ by

$$
\omega_{(1)}+u_{(0)}+\frac{\langle u \mid u\rangle}{2} \mathrm{id} .
$$

Since the spectrum of $u_{(0)}$ belongs to $(1 / n) \mathbb{Z}$, we obtain the following:

Lemma 4.5. If $\langle u \mid u\rangle \in(2 / n) \mathbb{Z}$, then the weights of $V^{(u)}$ belong to $(1 / n) \mathbb{Z}$.

Next, we consider the conformal weight of $V^{(u)}$. We now assume the following:

- $V$ is strongly regular;

- $V_{1}$ is semisimple; let $V_{1}=\bigoplus_{i=1}^{t} \mathfrak{g}_{i}$ be the decomposition of $V_{1}$ into the direct sum of simple ideals;

- the subVOA $U$ generated by $V_{1}$ is full, i.e., its conformal vector coincides with $\omega \in V$.

Note that the latter two assumptions hold for any (strongly regular) holomorphic VOA of $c=24$ if $V_{1}$ is neither $\{0\}$ nor abelian of rank 24 (see Proposition 2.2). By Proposition 2.1, $U$ is the tensor products of the simple affine VOAs $L_{\mathfrak{g}_{i}}\left(k_{i}, 0\right)$ associated with $\mathfrak{g}_{i}$ at positive level $k_{i}$. Then any irreducible $U$-module $M$ is isomorphic to $\bigotimes_{i=1}^{t} L_{\mathfrak{g}_{i}}\left(k_{i}, \lambda_{i}\right)$, where $L_{\mathfrak{g}_{i}}\left(k_{i}, \lambda_{i}\right)$ 's are irreducible $L_{\mathfrak{g}_{i}}\left(k_{i}, 0\right)$-modules with dominant integral weights $\lambda_{i}$ of $\mathfrak{g}_{i}$ at level $k_{i}$. Computing the conformal weight of the irreducible twisted module $M^{(u)}$, we obtain the following:

Lemma 4.6 (cf. [35]). If $u$ does not belong to the weight lattice of $V_{1}$, then the conformal weight of $V^{(u)}$ is positive.

Even if $u$ belongs to the weight lattice of $V_{1}$, we can also obtain a lower bound of the conformal weight of $V^{(u)}$ as follows:

(Step 1) Find all irreducible $U$-modules $M$ whose conformal weight is in $\mathbb{Z}_{\geq 2} \cup\{0\}$.

(Step 2) Calculate the conformal weight of $M^{(u)}$ for all $M$ in (Step 1).

Then the minimum of the conformal weights in (Step 2) gives a lower bound. By using this argument, we have confirmed in $[35,37]$ that the conformal weight of $V^{(u)}$ is at least 1 for some cases. 


\subsection{Dimension formulas and weight one Lie algebras}

In this subsection, we review certain dimension formulas for the weight one Lie algebras from [49], which was mentioned in [50]. This is useful for determining the weight one Lie algebra structure of a holomorphic VOA of $c=24$.

The following theorem is a variant of the dimension formula mentioned in $[50]$ :

Theorem 4.7 ([49, (4.10)]). Let $V$ be a strongly regular holomorphic VOA of $c=24$. Let $g$ be an automorphism of order $n$ satisfying the conditions (I) and (II) in Section 4.1. Assume that $n \in\{2,3,5,7,13\}$ and that the conformal weight of $V\left[g^{i}\right]$ is at least 1 for all $1 \leq i \leq n-1$. Then the following equation holds:

$$
\operatorname{dim} V_{1}+\operatorname{dim}\left(\widetilde{V}_{g}\right)_{1}=(n+1) \operatorname{dim} V_{1}^{g}+24
$$

\section{Remark 4.8.}

(1) More general formula is described in [50, 49, 23].

(2) For $n=2,3$, this theorem was also proved in [35, 37].

The key of the proof is that the character of $V^{g}$ is a modular function for the congruence group $\Gamma_{0}(n)$ [49, Proposition 4.8.2]. Hence, it can be described as a Laurent polynomial of a Hauptmodul of $\Gamma_{0}(n)$. By using the $S$-transformation, we obtain equations about the characters and trace functions of irreducible $V^{g}$-modules. Comparing some coefficients of the equations and using the assumption about the conformal weights of the irreducible twisted $V$-modules, we obtain this formula.

Let $V$ be a (strongly regular) holomorphic VOA of $c=24$ and $g$ an order $n$ automorphism of $V$ satisfying the conditions (I) and (II). Let $\widetilde{V}_{g}$ be the resulting holomorphic VOA obtained by the orbifold construction associated with $V$ and $g$. The following is a basic strategy in [35] to determine the weight one Lie algebra structure of $\widetilde{V}_{g}$ :

(1) Determine $d=\operatorname{dim}\left(\widetilde{V}_{g}\right)_{1}$ by using the dimension formula.

(2) List possible $d$-dimensional semisimple Lie algebra structures satisfying the equation in Proposition 2.2 with positive integral levels. 
There are only few possibilities by the argument above. Considering the embedding of Lie algebras and levels, we have only one possibility for the cases in [35]. Here, we also use the following fact:

- $\widetilde{V}_{g}$ has an automorphism $z$ of order $n$ such that $\left(\widetilde{V}_{g}\right)_{1}^{z}=\left(V^{g}\right)_{1}$.

This is related to the reverse orbifold construction, which will be discussed in Section 6.

\section{Constructions of 71 Holomorphic VOAs of Central Charge 24}

In this and the next sections, we will give a brief review on the recent progress towards the classification of strongly regular holomorphic VOAs of central charge 24 based on the strategy described in Section 3. In this section, we will discuss constructions of holomorphic VOAs that realize the Lie algebras in Schellekens' list. The main idea is to try to construct a new VOA from a known VOA by using the "Orbifold construction".

\subsection{Lattice vertex operator algebras}

We first recall the notion of lattice VOAs and review some of their properties. We use the standard notation for the lattice vertex operator algebra

$$
V_{L}=M(1) \otimes \mathbb{C}\{L\}
$$

associated with a positive-definite even lattice $L$ of rank $d$ [25]. Let $\mathfrak{h}=$ $\mathbb{C} \otimes_{\mathbb{Z}} L$ be an abelian Lie algebra. We extend the bilinear form $(\cdot \mid \cdot)$ to $\mathfrak{h}$ by $\mathbb{C}$-linearity. Let $\hat{\mathfrak{h}}=\mathfrak{h} \otimes \mathbb{C}\left[t, t^{-1}\right] \oplus \mathbb{C} k$ be the corresponding affine algebra, where $\mathbb{C} k$ is the 1 -dimensional center of $\hat{\mathfrak{h}}$. The subspace $M(1)=\mathbb{C}\left[\alpha_{i}(n) \mid 1 \leq\right.$ $i \leq d, n<0]$ for a basis $\left\{\alpha_{1}, \ldots, \alpha_{d}\right\}$ of $\mathfrak{h}$, where $\alpha(n)=\alpha \otimes t^{n}$, is the unique irreducible $\hat{\mathfrak{h}}$-module such that $\alpha(n) \cdot 1=0$ for all $\alpha \in \mathfrak{h}$ and $n$ nonnegative, and $k$ acts as the scalar 1. Also, $\mathbb{C}\{L\}=\operatorname{Span}\left\{e^{\beta} \mid \beta \in L\right\}$ is the twisted group algebra of the additive group $L$ such that $e^{\beta} e^{\alpha}=(-1)^{(\alpha \mid \beta)} e^{\alpha} e^{\beta}$ for any $\alpha, \beta \in L$. The vacuum vector $\mathbb{1}$ of $V_{L}$ is $1 \otimes e^{0}$ and the conformal vector $\omega$ is $\frac{1}{2} \sum_{i=1}^{d} \beta_{i}(-1)^{2} \cdot \mathbb{1}$, where $\left\{\beta_{1}, \ldots, \beta_{d}\right\}$ is an orthonormal basis of $\mathfrak{h}$. For the explicit definition of the corresponding vertex operators, we will refer 
to [25] for details. We also note that $V_{L}$ is strongly regular and the central charge of $V_{L}$ is equal to $d$, the rank of $L$.

Let $L^{*}=\left\{v \in \mathbb{R} \otimes_{\mathbb{Z}} L \mid(v \mid L) \subset \mathbb{Z}\right\}$ be the dual lattice of $L$. For $\alpha+L \in L^{*} / L$, denote $V_{\alpha+L}=M(1) \otimes \mathbb{C}\{\alpha+L\}$, where $\mathbb{C}\{\alpha+L\}=$ $\operatorname{Span}\left\{e^{\beta} \mid \beta \in \alpha+L\right\} \subset \mathbb{C}\left\{L^{*}\right\}$. Then $V_{\alpha+L}$ is an irreducible $V_{L}$-module [25]. It was proved in [8] that any irreducible $V_{L}$-module is isomorphic to $V_{\alpha+L}$ for some $\alpha+L \in L^{*} / L$. In particular, we have the following result.

Theorem 5.1. Let $L$ be an even lattice of rank d. If $L$ is unimodular, i.e., $L^{*}=L$, then $V_{L}$ is a strongly regular holomorphic VOA of central charge $d$.

Even unimodular lattices of rank 24 were classified by Niemeier ([51]); there are exactly 24 such lattices and their isometry types are determined by the structures of their norm 2 vectors, which are also known as the root systems in the literature.

Proposition 5.2. There exist holomorphic VOAs of central charge 24 whose weight one Lie algebras have the following 24 types:

$A_{1,1}^{24}, \quad A_{2,1}^{12}, \quad A_{3,1}^{8}, \quad A_{4,1}^{6}, \quad A_{5,1}^{4} D_{4,1}, \quad D_{4,1}^{6}, \quad A_{6,1}^{4}, \quad A_{7,1}^{2} D_{5,1}^{2}$, $A_{8,1}^{3}, \quad A_{9,1}^{2} D_{6,1}, \quad D_{6,1}^{4}, \quad E_{6,1}^{4}, \quad A_{11,1} D_{7,1} E_{6,1}, \quad A_{12,1}^{2}, \quad D_{8,1}^{3}, \quad A_{15,1} D_{9,1}$, $A_{17,1} E_{7,1}, D_{10,1} E_{7,1}^{2}, D_{12,1}^{2}, A_{24,1}, D_{16,1} E_{8,1}, \quad E_{8,1}^{3}, \quad D_{24,1}, U(1)^{24}$,

where $U(1)$ is a 1-dimensional abelian Lie algebra.

Remark 5.3. For even lattices $L$ and $M$, the VOAs $V_{L}$ and $V_{M}$ are isomorphic if and only if the lattices $L$ and $M$ are isometric. Hence there exist exactly 24 holomorphic lattice VOAs of central charge 24 .

\subsection{Irreducible twisted modules for lattice VOAs}

In this subsection, we review a construction of irreducible twisted $V_{L^{-}}$ modules, which will be used to apply the $\mathbb{Z}_{n}$-orbifold construction to $V_{L}$.

Let $L$ be an even unimodular lattice and $O(L)$ the isometry group of $L$. For $g \in O(L)$, set $L^{g}=\{v \in L \mid g(v)=v\}$ and let $P_{0}^{g}$ be the orthogonal projection from $\mathbb{Q} \otimes_{\mathbb{Z}} L$ to $\mathbb{Q} \otimes_{\mathbb{Z}} L^{g}$. Let $\hat{L}=\left\{ \pm e^{\alpha} \mid \alpha \in L\right\}$ be a central extension of $L$ by $\langle-1\rangle$ with the commutator relation $e^{\beta} e^{\alpha}=(-1)^{(\alpha \mid \beta)} e^{\alpha} e^{\beta}$. 
Let Aut $(\hat{L})$ be the set of all group automorphisms of $\hat{L}$. For $\varphi \in \operatorname{Aut}(\hat{L})$, we define the group automorphism $\bar{\varphi}$ of $L$ by $\varphi\left(e^{\alpha}\right) \in\left\{ \pm e^{\bar{\varphi}(\alpha)}\right\}, \alpha \in L$. Set

$$
O(\hat{L})=\{\varphi \in \operatorname{Aut}(\hat{L}) \mid \bar{\varphi} \in O(L)\} .
$$

Then $O(\hat{L})$ acts on $V_{L}$ as an automorphism group ([25]).

Let $g \in O(L)$ be of order $n$. We call $\varphi \in O(\hat{L})$ a lift of $g$ if $\bar{\varphi}=g$. A lift $\phi_{g} \in O(\hat{L})$ of $g$ is called standard if $\phi_{g}\left(e^{\alpha}\right)=e^{\alpha}$ for $\alpha \in L^{g}$. Note that a standard lift $\phi_{g}$ always exists ([41]) and its order is $n$ or $2 n$ (see $[22,49]$ for detail). Then $V_{L}$ has a unique irreducible $\phi_{g}$-twisted $V_{L}$-module, up to isomorphism ([13]). Such a module $V_{L}\left[\phi_{g}\right]$ was constructed in $[41,11]$ explicitly; as a vector space,

$$
V_{L}\left[\phi_{g}\right] \cong M(1)[g] \otimes \mathbb{C}\left[P_{0}^{g}(L)\right] \otimes T,
$$

where $M(1)[g]$ is the " $g$-twisted" free bosonic space, $\mathbb{C}\left[P_{0}^{g}(L)\right]$ is the group algebra of $P_{0}^{g}(L)$ and $T$ is an irreducible module for a certain " $g$-twisted" central extension of $L$. (see [41, Propositions 6.1 and 6.2] and [11, Remark 4.2] for detail). Recall that

$$
\operatorname{dim} T=\left|L_{g} /(1-g) L\right|^{1 / 2}
$$

and that the weight $\rho_{g}$ of $T$ is given by

$$
\rho_{g}:=\frac{1}{4 n^{2}} \sum_{j=1}^{n-1} j(n-j) \operatorname{dim} \mathfrak{h}_{(j)},
$$

where $L_{g}=\left\{v \in L \mid\left(v \mid L^{g}\right)=0\right\}$ and $\mathfrak{h}_{(j)}=\left\{v \in \mathfrak{h} \mid g(v)=e^{(2 j \pi \sqrt{-1}) / n} v\right\}$. The weight of an element $v_{1}\left(-n_{1}\right) \ldots v_{s}\left(-n_{s}\right) \otimes e^{\alpha} \otimes t \in V_{L}\left[\phi_{g}\right]$ is given by

$$
\sum_{i=1}^{s} n_{i}+\frac{(\alpha \mid \alpha)}{2}+\rho_{g}
$$

where $v_{1}\left(-n_{1}\right) \ldots v_{s}\left(-n_{s}\right) \in M(1)[g], e^{\alpha} \in \mathbb{C}\left[P_{0}^{g}(L)\right]$ and $t \in T$. Note that $n_{i} \in(1 / n) \mathbb{Z}_{>0}$ and that the conformal weight of $V_{L}\left[\phi_{g}\right]$ is $\rho_{g}$. 


\section{3. $\mathbb{Z}_{2}$-orbifold construction associated with a lift of the -1-isometry}

The original construction of the moonshine VOA $V^{\natural}$ is done by applying the $\mathbb{Z}_{2}$-orbifold construction to the Leech lattice VOA and an order 2 lift of the -1-isometry [25]. This construction was later generalized to other Niemeier lattice VOAs by [7]. Now let us recall the construction.

Let $N$ be a Niemeier lattice, a positive-definite even unimodular lattice of rank 24, and $V_{N}$ the lattice VOA associated with $N$. Let $\theta$ be an order 2 automorphism of $V_{N}$ such that $\theta\left(e^{\alpha}\right)=\lambda_{\alpha} e^{-\alpha}, \lambda_{\alpha} \in\{ \pm 1\}$ for all $\alpha \in N$, i.e., $\theta$ is a lift of the -1 -isometry of $N$. Let $V_{N}[\theta]$ be the irreducible $\theta$-twisted $V_{L}$-module. Then, by (5.1), the conformal weight of $V_{N}[\theta]$ is $3 / 2$. Hence $V_{N}[\theta]$ satisfies the conditions (I) and (II) for the orbifold construction in Section 4.1. Applying the $\mathbb{Z}_{2}$-orbifold construction to $V_{N}$ and $\theta$, we obtain a strongly regular holomorphic VOA $\left(\widetilde{V_{N}}\right)_{\theta}$ of central charge 24 .

Since the conformal weight of $V_{N}[\theta]$ is $3 / 2$, we have $\left(\left(\widetilde{V_{N}}\right)_{\theta}\right)_{1}=\{v \in$ $\left.\left(V_{N}\right)_{1} \mid \theta(v)=v\right\}$. The weight one Lie algebra of $\left(\widetilde{V}_{N}\right)_{\theta}$ is determined in $[25,7]$, and there are 9 cases that the holomorphic $\operatorname{VOA}\left(\widetilde{V_{N}}\right)_{\theta}$ is again isomorphic to a Niemeier lattice VOA. The remaining 15 holomorphic VOAs are non-isomorphic to each other since their weight one Lie algebras are nonisomorphic.

Proposition 5.4 ([25, 7]). There exist holomorphic VOAs of central charge 24 whose weight one Lie algebras have the following 15 types:

$$
\begin{aligned}
& A_{1,4}^{12}, A_{1,2}^{16}, \quad C_{2,2}^{6}, \quad A_{3,2}^{4} A_{1,1}^{4}, \quad B_{3,2}^{4}, \quad D_{4,2}^{2} C_{2,1}^{4}, B_{4,2}^{3}, D_{5,2}^{2} A_{3,1}^{2} \text {, } \\
& C_{4,1}^{4}, D_{6,2} C_{4,1} B_{3,1}^{2}, B_{6,2}^{2}, D_{8,2} B_{4,1}^{2}, D_{9,2} A_{7,1}, B_{12,2}, \quad 0 .
\end{aligned}
$$

Remark 5.5. Let $\mathcal{C}$ be a doubly even self-dual binary code of length 24 .

Define

$$
\begin{aligned}
A(\mathcal{C}) & =\frac{1}{\sqrt{2}}\left\{\left(x_{1}, \ldots, x_{24}\right) \in \mathbb{Z}^{24} \mid\left(x_{i} \quad(\bmod 2)\right) \in \mathcal{C}\right\}, \\
C(\mathcal{C}) & =\{v \in A(\mathcal{C}) \mid(v \mid(1,1, \ldots, 1)) \in \sqrt{2} \mathbb{Z}\} \oplus \mathbb{Z} \frac{1}{2 \sqrt{2}}(-3,1,1 \ldots, 1) .
\end{aligned}
$$

Then $A(\mathcal{C})$ and $C(\mathcal{C})$ are even unimodular [6] and their rank are 24. It was shown in $[7$, Proposition 7.3$]$ that $V_{C(\mathcal{C})} \cong\left(\widetilde{V_{A(\mathcal{C})}}\right)_{\theta}$. Note that there exist exactly 9 doubly even self-dual binary codes of length 24 , up to equivalence. 


\subsection{Framed vertex operator algebras}

Next we discuss constructions for 17 holomorphic VOAs of central charge 24 as framed VOAs from [31, 33]. For the fundamental results about framed VOAs, see [9, 45, 40].

Let $L(1 / 2,0)$ be the simple Virasoro VOA of central charge $1 / 2$ and let $L(1 / 2, h), h \in\{1 / 2,1 / 16\}$, be the irreducible $L(1 / 2,0)$-module with conformal weight $h$. For the explicit construction of Virasoro VOAs and their modules, we refer to [26] for details. It is well-known that $L(1 / 2,0)$ is strongly regular and has exactly three irreducible modules, namely, $L(1 / 2,0), L(1 / 2,1 / 2)$, and $L(1 / 2,1 / 16)$. Moreover, the fusion products are known [19]:

$$
\begin{aligned}
L(1 / 2,1 / 2) \otimes L(1 / 2,1 / 2) & =L(1 / 2,0), \\
L(1 / 2,1 / 2) \otimes L(1 / 2,1 / 16) & =L(1 / 2,1 / 16), \\
L(1 / 2,1 / 16) \otimes L(1 / 2,1 / 16) & =L(1 / 2,0) \oplus L(1 / 2,1 / 2) .
\end{aligned}
$$

Definition 5.6 ([9]). A simple VOA $V$ of central charge $n \in \mathbb{Z} / 2$ is said to be framed if it contains a full subVOA isomorphic to the tensor product VOA $L(1 / 2,0)^{\otimes 2 n}$. Such a full subVOA is called a Virasoro frame of $V$.

Given a framed VOA $V$ of central charge $n / 2$ with a Virasoro frame $F$, one can associate two binary codes $C$ and $D$ of length $n$ with $V$ and $F$ by the following way:

Since $F\left(\cong L(1 / 2,0)^{\otimes n}\right)$ is rational, $V$ is completely reducible as an $F$ module. That is,

$$
V \cong \bigoplus_{h_{i} \in\{0,1 / 2,1 / 16\}} m_{h_{1}, \ldots, h_{n}} L\left(1 / 2, h_{1}\right) \otimes \cdots \otimes L\left(1 / 2, h_{n}\right)
$$

where the nonnegative integer $m_{h_{1}, \ldots, h_{n}}$ is the multiplicity of $L\left(1 / 2, h_{1}\right) \otimes \cdots \otimes$ $L\left(1 / 2, h_{n}\right)$ in $V$ and all $m_{h_{1}, \ldots, h_{n}}$ 's are finite.

Definition 5.7. Let $M \cong L\left(1 / 2, h_{1}\right) \otimes \cdots \otimes L\left(1 / 2, h_{n}\right)$ be an irreducible $F$ module. We define $\tau(M)$ of $M$ as the binary word $\beta=\left(\beta_{1}, \ldots, \beta_{n}\right) \in \mathbb{Z}_{2}^{n}$ such that

$$
\beta_{i}= \begin{cases}0 & \text { if } h_{i}=0 \text { or } 1 / 2 \\ 1 & \text { if } h_{i}=1 / 16\end{cases}
$$


For any $\beta \in \mathbb{Z}_{2}^{n}$, let $V^{\beta}$ be the sum of all irreducible $F$-submodules $M$ of $V$ such that $\tau(M)=\beta$.

Definition 5.8. Let $D:=\left\{\beta \in \mathbb{Z}_{2}^{n} \mid V^{\beta} \neq 0\right\}$. Then $D$ is a linear subcode of $\mathbb{Z}_{2}^{n}$, and it is called the $1 / 16$-code of $V$ with respect to $F$.

Now we identify the set $\{0,1\}$ with $\mathbb{Z}_{2}$. For $\alpha=\left(\alpha_{1}, \ldots, \alpha_{n}\right) \in \mathbb{Z}_{2}^{n}$, define

$$
m_{\alpha}=m_{\alpha_{1} / 2, \ldots, \alpha_{n} / 2} \quad \text { and } \quad M_{\alpha}=L\left(1 / 2, \alpha_{1} / 2\right) \otimes \cdots \otimes L\left(1 / 2, \alpha_{n} / 2\right) .
$$

Then $V^{0}$ is given by

$$
V^{0}=\bigoplus_{\alpha \in \mathbb{Z}_{2}^{n}} m_{\alpha} M_{\alpha}
$$

It is proved in [19] that $m_{h_{1}, \ldots, h_{n}}$ is at most 1 if all $h_{i}$ are different from $1 / 16$.

Definition 5.9. Let $C:=\left\{\alpha \in \mathbb{Z}_{2}^{n} \mid m_{\alpha} \neq 0\right\}$. Then $C$ is also a linear code and is called the $1 / 2$-code of $V$ with respect to $F$.

Summarizing, there exists a pair of binary codes $(C, D)$ of length $n$ such that

$$
V=\bigoplus_{\beta \in D} V^{\beta} \quad \text { and } \quad V^{0}=\bigoplus_{\alpha \in C} M_{\alpha}
$$

The codes $(C, D)$ are called the structure codes of a framed VOA $V$ associated with the frame $F$. Note that the structure codes depend on the choice of a Virasoro frame.

The next lemma follows from the integral condition on the weights of $V:$

Lemma 5.10. The $1 / 16^{-c o d e} D$ is triply even, i.e., $\operatorname{wt}(\alpha) \equiv 0 \bmod 8$ for all $\alpha \in D$ and the $1 / 2$-code $C$ is even. Moreover, $D<C^{\perp}=\left\{\beta \in \mathbb{Z}_{2}^{n} \mid(\alpha, \beta)=\right.$ 0 for all $\alpha \in C\}$, where $(\cdot, \cdot)$ is the standard inner product of $\mathbb{Z}_{2}^{n}$.

The following theorems are also well-known:

Theorem 5.11 ([9, Theorem 2.9] and [45, Theorem 6.1]). Let $V$ be a framed $V O A$ with the structure codes $(C, D)$. Then, $V$ is holomorphic if and only if $C=D^{\perp}$. 
Theorem 5.12 ([40, Theorem 10]). Let $D$ be a linear binary code of length $16 k, k \in \mathbb{Z}_{>0}$. Assume that $D$ is triply even and contains the all-one vector. Then there exists a holomorphic framed VOA of central charge $8 k$ with the structure codes $\left(D^{\perp}, D\right)$.

Remark 5.13. In the proof of Theorem 5.12, the $\mathbb{Z}_{2}$-orbifold constructions associated with holomorphic framed VOAs and certain order 2 automorphisms, often called Miyamoto involutions [44], play important roles. It turns out that any holomorphic framed VOA can be obtained by several consecutive $\mathbb{Z}_{2}$-orbifold constructions from a holomorphic lattice VOA.

Theorem 5.12 also suggests a method for classifying all holomorphic framed VOAs of central charge 24 as follows:

(i) Classify all triply even codes of length 48 containing the all-one vector.

(ii) For each code $D$ in (i), classify all possible holomorphic framed VOA structures with structure codes $\left(D^{\perp}, D\right)$.

A classification of all triply even codes of length 48 has been obtained in [1]. By the results in [1], all possible Lie algebra structures for the weight one Lie algebras of holomorphic framed VOAs of central charge 24 were determined in $[31,33]$. In particular, we have the following theorem.

Theorem $5.14([31,33])$. There exist holomorphic framed VOAs of central charge 24 whose weight one Lie algebras have the following 17 types:

$A_{3,4}^{3} A_{1,2}, \quad D_{5,8} A_{1,2}, \quad D_{4,4} A_{2,2}^{4}, \quad A_{7,4} A_{1,1}^{3}, \quad D_{5,4} C_{3,2} A_{1,1}^{2}, C_{4,2} A_{4,2}^{2}$, $A_{5,2}^{2} C_{2,1} A_{2,1}^{2}, A_{7,2} C_{3,1}^{2} A_{3,1}, C_{7,2} A_{3,1}, \quad A_{8,2} F_{4,2}, A_{9,2} A_{4,1} B_{3,1}, E_{6,2} C_{5,1} A_{5,1}$, $C_{6,1} B_{4,1}, \quad C_{8,1} F_{4,1}^{2}, \quad E_{7,2} B_{5,1} F_{4,1}, C_{10,1} B_{6,1}, E_{8,2} B_{8,2}$.

Remark 5.15. Any Niemeier lattice $N$ contains an orthogonal basis consisting of norm 4 vectors. Such an orthogonal basis defines a Virasoro frame $F$ of the Niemeier lattice VOA $V_{N}$ (cf. $[9,(3.1)]$ ), and hence $V_{N}$ is framed. Since $F$ is fixed by $\theta$, the holomorphic $\operatorname{VOA}\left(\widetilde{V}_{N}\right)_{\theta}$ contains $F$, which shows that $\left(\widetilde{V}_{N}\right)_{\theta}$ is also framed. Thus the 39 holomorphic VOAs in Propositions 5.2 and 5.4 are also framed.

Remark 5.16. Other constructions of holomorphic framed VOAs are studied independently. In [28], holomorphic VOAs of central charge 24 whose weight one Lie algebra has type $A_{8,2} F_{4,2}, C_{4,2} A_{4,2}^{2}$ and $D_{4,4} A_{2,2}^{4}$ were constructed as simple current extensions of $V_{\sqrt{2} E_{8} \oplus \sqrt{2} D_{4}} \otimes V_{\sqrt{2} D_{12}^{+}}^{+}$. In [10], a 
holomorphic VOA of central charge 24 whose weight one Lie algebra has type $A_{9,2} A_{4,1} B_{3,1}$ was constructed by using mirror extension.

A complete answer to (ii) was also established in [34] as follows:

Theorem 5.17 ([34]). The isomorphism class of a holomorphic framed VOA of central charge 24 is uniquely determined by the Lie algebra structure of its weight one subspace. In particular, there exist exactly 56 holomorphic framed VOAs of central charge 24, up to isomorphism.

Remark 5.18. For any holomorphic framed VOA of central charge 24, the levels of simple ideals of its weight one Lie algebra are power of two. Conversely, by comparing with the list of Lie algebras in [53], we see that except for one case $E_{6,4} C_{2,1} A_{2,1}$, all other Lie algebras in [53] can be obtained from holomorphic framed VOAs if the levels are powers of two. This exceptional case has recently obtained by a $\mathbb{Z}_{4}$-orbifold construction from a lattice VOA in $[22,49]$.

\section{5. $\mathbb{Z}_{n}$-orbifold constructions associated with the Niemeier lattice}

\section{VOAs}

In this subsection, we review $\mathbb{Z}_{n}$-orbifold constructions associated with the Niemeier lattice VOAs, which provides explicit construction of many holomorphic VOAs of central charge 24. See Section 4 for $\mathbb{Z}_{n}$-orbifold constructions.

\subsection{1. $\mathbb{Z}_{3}$-orbifold construction}

Let $N$ be a Niemeier lattice. Let $g$ be an order 3 isometry of $N$ such that the rank of $N^{g}$ is a multiple of 6 . Since the order of $g$ is odd, a standard lift $\phi_{g}$ has order 3 ([22, Proposition 7.3]). The assumption on the rank of $N^{g}$ guarantees that the conformal weight $\rho_{g} \in(1 / 3) \mathbb{Z}_{>0}$ (see (5.1)). In [46], the $\mathbb{Z}_{3}$-orbifold construction associated with $V_{N}$ and $\phi_{g}$ was established, and as an application, a holomorphic VOA of central charge 24 whose weight one Lie algebra has type $E_{6,3} G_{2,1}^{3}$ was constructed. By considering all Niemeier lattices and suitable order 3 isometries of the Niemeier lattices, two more holomorphic VOAs of central charge 24 were constructed in [52] (cf. [29]); their weight one Lie algebras have the type $A_{2,3}^{6}$ and $A_{5,3} D_{4,3} A_{1,1}^{3}$. 
Theorem 5.19 ([46, 52]). There exist holomorphic VOAs of central charge 24 whose weight one Lie algebras have the following 3 types: $A_{2,3}^{6}, A_{5,3} D_{4,3} A_{1,1}^{3}$ and $E_{6,3} G_{2,1}^{3}$.

Remark 5.20. In [46], a holomorphic VOA of central charge 24 with trivial weight 1 space was constructed by applying the $\mathbb{Z}_{3}$-orbifold construction to the Leech lattice VOA and a fixed-point free isometry of order 3. It was first announced in [14] that it is isomorphic to $V^{\natural}$, which was recently verified in $[4]$.

\subsection{2. $\mathbb{Z}_{n}$-orbifold construction for $n \geq 4$}

Inspired by Miyamoto's $\mathbb{Z}_{3}$-orbifold construction, a $\mathbb{Z}_{n}$-orbifold construction was established in $[22,49]$ (see Section 4.1). Applying the $\mathbb{Z}_{n}$-orbifold construction to Niemeier lattice VOAs and some finite order isometries of the Niemeier lattices, they obtained the following result.

Theorem 5.21 ([22, 49]). There exist holomorphic VOAs of central charge 24 whose weight one Lie algebras have the following 5 types: $A_{2,1} B_{2,1} E_{6,4}$, $A_{4,5}^{2}, A_{2,6} D_{4,12}, A_{1,1} C_{5,3} G_{2,2}$ and $C_{4,10}$.

Remark 5.22. Many holomorphic framed VOAs in Theorem 5.14 can be constructed by $\mathbb{Z}_{2}, \mathbb{Z}_{4}$ or $\mathbb{Z}_{8}$-orbifold construction associated with the Niemeier lattice VOAs, also.

\subsection{Orbifold construction associated with inner automorphisms}

In [35], the orbifold construction associated with inner automorphisms was studied. In particular, five holomorphic VOAs of central charge 24 were constructed. In this subsection, we review the constructions.

In [35], the orbifold construction associated with an inner automorphism $\sigma_{u}$ was studied based on the analysis in [43] and [12]. An advantage for using an inner automorphism is that $\sigma_{u}$ is defined globally on the whole VOA $V$. Moreover, an explicit construction of the irreducible $\sigma_{u}$-twisted module was obtained in [43] by using the $\Delta$-operator (see Section 4.2). Therefore, we can apply the orbifold construction to non-lattice type VOA, also. By the definition of the twisted modules, it is relatively easy to obtain a necessary 
and sufficient condition on $u$ so that the conformal weights of the irreducible twisted $\sigma_{u}^{i}$-module belong to $(1 / n) \mathbb{Z}_{>0}$ (see Lemmas 4.5 and 4.6 ).

By choosing the holomorphic VOA $V$ and its inner automorphism $\sigma_{u}$ of order 2 carefully, several new holomorphic VOAs of central charge 24 were constructed in [35] by applying the $\mathbb{Z}_{2}$-orbifold construction to $V$ and $\sigma_{u}$. The Lie algebra structures of $V_{1},\left(V^{\sigma_{u}}\right)_{1}$ and $\widetilde{V}_{1}$ were summarized in Table 1, where $\widetilde{V}$ denotes the resulting VOA $\widetilde{V}_{\sigma_{u}}$ obtained by the orbifold construction and $V^{\sigma_{u}}$ is the set of fixed-points of $\sigma_{u}$.

Table 1: Lie algebra structures of $V_{1},\left(V^{\sigma_{u}}\right)_{1}$ and $\widetilde{V}_{1}$.

\begin{tabular}{|c|c|c|}
\hline (Original) Lie algebra $V_{1}$ & (Fixed point) Lie subalgebra $\left(V^{\sigma_{u}}\right)_{1}$ & $($ New $)$ Lie algebra $\widetilde{V}_{1}$ \\
\hline \hline$E_{6,3} G_{2,1}^{3}$ & $D_{5,3} A_{1,1}^{2} A_{1,3}^{2} G_{2,1} U(1)$ & $D_{7,3} A_{3,1} G_{2,1}$ \\
\hline$D_{7,3} A_{3,1} G_{2,1}$ & $D_{6,3} A_{3,1} A_{1,1} A_{1,3} U(1)$ & $E_{7,3} A_{5,1}$ \\
\hline$E_{7,3} A_{5,1}$ & $A_{7,3} A_{2,1}^{2} U(1)$ & $A_{8,3} A_{2,1}^{2}$ \\
\hline$C_{5,3} G_{2,2} A_{1,1}$ & $A_{4,6} A_{1,6} A_{1,2} U(1)^{2}$ & $A_{5,6} C_{2,3} A_{1,2}$ \\
\hline$A_{4,5}^{2}$ & $A_{3,5}^{2} U(1)^{2}$ & $D_{6,5} A_{1,1}^{2}$ \\
\hline
\end{tabular}

Theorem 5.23 ([35]). There exist holomorphic VOAs of central charge 24 whose weight one Lie algebras have the following 5 types: $D_{7,3} A_{3,1} G_{2,1}$, $E_{7,3} A_{5,1}, A_{8,3} A_{2,1}^{2}, D_{6,5} A_{1,1}^{2}$ and $A_{5,6} C_{2,3} A_{1,2}$.

In the determination of the Lie algebra structure of the resulting VOA, we use the argument as described in Section 4.3. In particular, the dimension formula in Theorem 4.7 plays a crucial role in our calculation.

\subsection{Orbifold construction associated with non-standard lifts}

In this subsection, we discuss a construction of a holomorphic VOA of central charge 24 whose weight one Lie algebra has the type $A_{6,7}$ from [36].

Since the level of $A_{6,7}$ is 7 , it is expected that such a VOA can be constructed by a $\mathbb{Z}_{7}$-orbifold construction. It is indeed correct; however, the choice of the automorphism is tricky. It was shown in [36] that a holomorphic VOA $V$ of central charge 24 with $V_{1}=A_{6,7}$ can be constructed by applying the $\mathbb{Z}_{7}$-orbifold construction to the Leech lattice VOA $V_{\Lambda}$ and an 
order 7 automorphism of $V_{\Lambda}$ but the desired automorphism is the product of a standard lift of an order 7 isometry of $\Lambda$ and an order 7 inner automorphism of $V_{\Lambda}$. An explicit construction of the irreducible twisted $V_{\Lambda}$-module is also obtained in [36] by combining the explicit construction of the twisted $V_{\Lambda}$-modules for an isometry of $\Lambda$ (see Section 5.2) and the modification by Li's $\Delta$-operator (see Section 4.2). Moreover, it was proved that the weight one subspace of the resulting orbifold VOA has dimension 48 and it is a simple Lie algebra of type $A_{6}$.

Theorem 5.24 ([36]). There exists a holomorphic VOA of central charge 24 whose weight one Lie algebra has the type $A_{6,7}$.

\subsection{A holomorphic VOA $V$ with $V_{1}=F_{4,6} A_{2,2}$}

Finally, we discuss a construction of a strongly regular holomorphic VOA of central charge 24 whose weight one Lie algebra has the type $F_{4,6} A_{2,2}$. Such a VOA is constructed in [32] by applying a $\mathbb{Z}_{2}$-orbifold construction to a holomorphic VOA $U$ with the weight one Lie algebra $A_{8,3} A_{2,1}^{2}$ and a suitable automorphism $g$ of order 2. Although it is also an orbifold construction, there are some fundamental differences between this case and the previous constructions for the other cases. In this construction, the fixed points of the automorphism $g$ on $U_{1}=A_{8,3} A_{2,1}^{2}$ have the type $B_{4,6} A_{2,2}$. Therefore, $\left.g\right|_{U_{1}}$ is an outer automorphism of the Lie algebra $U_{1}$. In general, it is very difficult to determine if an outer automorphism of Lie algebra $U_{1}$ can be extended to an automorphism of the whole VOA. An explicit construction of the corresponding twisted module is also missing for such an automorphism.

As we mentioned in Section 5.6, a construction of a holomorphic VOA $U$ of central charge 24 with $U_{1}=A_{8,3} A_{2,1}^{2}$ is obtained in [35] by using orbifold construction. On the other hand, there is an alternative construction based on mirror extensions of VOAs $[56,10]$. This alternative construction is first obtained by $\mathrm{Xu}[56]$ in terms of conformal nets and is proposed in [10] in VOA setting. Using mirror extensions and the theory of modular invariants, it was shown in [32] that the VOA structure of a holomorphic VOA $U$ of central charge 24 with $U_{1}=A_{8,3} A_{2,1}^{2}$ is unique, up to isomorphism. Moreover, by generalizing a result of [54], a sufficient condition for extending an automorphism of a subVOA to the whole VOA is obtained. By these two facts, it was shown that if $U$ is a strongly regular holomorphic VOA of 
central charge 24 such that $U_{1}=A_{8,3} A_{2,1}^{2}$, then there exists an involution $g \in \operatorname{Aut}(U)$ such that $U_{1}^{g}$ is a Lie algebra of type $B_{4,6} A_{2,2}$ [32, Corollary 4.20]. The conformal weight of the unique irreducible $g$-twisted $U$-module $U[g]$ is also determined using the explicit action of $g$ on $U_{1}$. As a consequence, the following result is proved in [32].

Theorem 5.25 ([32]). There exists a holomorphic VOA of central charge 24 whose weight one Lie algebra has the type $F_{4,6} A_{2,2}$.

By the results in Sections 5, it is known that all 71 Lie algebras in Schellekens' list can be realized as the weight one Lie algebra of some holomorphic VOA of central charge 24.

\section{Uniqueness of Holomorphic VOAs of Central Charge 24}

In Section 5, we have seen the constructions of 71 holomorphic VOAs of central charge 24. In order to complete the classification, we have to deal with the uniqueness problem, which is the main topic of this section. In particular, we will review a technique which we call "reverse orbifold construction" and discuss its application towards the uniqueness problem.

\subsection{Known uniqueness results}

In this subsection, we review the known results on the uniqueness of holomorphic VOAs of central charge 24. First, we recall a characterization of Niemeier lattice VOAs.

Theorem 6.1 ([16, Corollary 1.4]). Let $V$ be a strongly regular holomorphic $V O A$ of central charge 24. If the Lie rank of $V_{1}$ is 24, then $V$ is isomorphic to a Niemeier lattice VOA.

By this theorem, the uniqueness of holomorphic VOAs are established for the 24 types of Lie algebras in Theorem 5.2.

It is also well-known that the simple affine VOAs of type $A_{1,2}, B_{8,1}$ and $E_{8,2}$ are framed (cf. [31]). Hence, as a corollary of Theorem 5.17, we obtain the following: 
Corollary 6.2. Let $V$ be a strongly regular holomorphic VOA of central charge 24. Assume that the weight one Lie algebra $V_{1}$ has type $A_{1,2}^{16}$ or $B_{8,1} E_{8,2}$. Then $V$ is framed. In particular, the VOA structure of $V$ is unique up to isomorphism.

As we discussed in Section 5.8, there is also a uniqueness result for Lie algebra of the type $A_{8,3} A_{2,1}^{2}$ :

Proposition 6.3. A strongly regular holomorphic VOA structure of central charge 24 is unique if the weight one Lie algebra has the type $A_{8,3} A_{2,1}^{2}$.

\subsection{Reverse orbifold construction and its application}

In this subsection, we review the reverse orbifold construction from [37] (cf. $[22,49])$ and its application.

Let $V$ be a holomorphic VOA and let $g$ be an order $n$ automorphism of $V$ satisfying the conditions (I) and (II) in Section 4.1. Let $W=\widetilde{V}_{g}$ be the resulting holomorphic VOA by applying $\mathbb{Z}_{n}$-orbifold construction to $V$ and $g$. Let $h$ be an order $n$ automorphism of $W$ associated with the $\mathbb{Z}_{n}$-grading of $W$. Then $W^{h}=V^{g}$ and, for $1 \leq i \leq n-1$, the irreducible $h^{i}$-twisted $W$-module is a direct sum of irreducible $V^{g}$-modules. Hence $h$ also satisfies (I) and (II). By the uniqueness of the resulting holomorphic VOA by the orbifold construction, we obtain the following:

Corollary 6.4 ([37], see also $[22,49])$. The VOA $\widetilde{W}_{h}$ is isomorphic to $V$.

We call this procedure the reverse orbifold construction, which is called the inverse orbifold in $[22,49]$. By using this corollary, we can prove the following theorem about the uniqueness of holomorphic VOAs.

Theorem 6.5. Let $\mathfrak{g}$ be a Lie algebra and $\mathfrak{p}$ a subalgebra of $\mathfrak{g}$. Let $n \in$ $\mathbb{Z}_{>0}$ and let $W$ be a strongly regular holomorphic VOA of central charge $c$. Assume that for any strongly regular holomorphic VOA $V$ of central charge $c$ whose weight one Lie algebra is $\mathfrak{g}$, there exists $g \in \operatorname{Aut}(V)$ of order $n$ satisfying the conditions (I) and (II) such that the following conditions hold:

(a) $\mathfrak{g}^{g} \cong \mathfrak{p}$;

(b) $\widetilde{V}_{g}$ is isomorphic to $W$. 
In addition, we assume that any automorphism $\varphi \in \operatorname{Aut}(W)$ of order $n$ satisfying (I) and (II) and the conditions (A) and (B) below belongs to a unique conjugacy class in $\operatorname{Aut}(W)$ :

(A) $\left(W^{\varphi}\right)_{1}$ is isomorphic to $\mathfrak{p}$;

(B) $\left(\widetilde{W}_{\varphi}\right)_{1}$ is isomorphic to $\mathfrak{g}$.

Then any strongly regular holomorphic VOA of central charge $c$ with weight one Lie algebra $\mathfrak{g}$ is isomorphic to $\widetilde{W}_{\varphi}$. In particular, such a holomorphic $V O A$ is unique up to isomorphism.

Proof. Let $V$ be a strongly regular holomorphic VOA of central charge $c$ such that $V_{1} \cong \mathfrak{g}$. By (b), $\widetilde{V}_{g} \cong W$; we identify $\widetilde{V}_{g}$ with $W$. Let $h$ be an order $n$ automorphism of $W$ associated with the $\mathbb{Z}_{n}$-grading of $W$ as the extension of $V^{g}$. Then $\left(W^{h}\right)_{1} \cong V_{1}^{g} \cong \mathfrak{p}$ by (a). By Corollary 6.4, $\widetilde{W}_{h} \cong V$. Hence $\left(\widetilde{W}_{h}\right)_{1} \cong V_{1}=\mathfrak{g}$. Since $h$ satisfies $(\mathrm{A})$ and $(\mathrm{B})$, it is conjugate to $\varphi$. By Remark $4.3(2), V \cong \widetilde{W}_{h} \cong \widetilde{W}_{\varphi}$.

Remark 6.6. In Theorem 6.5, the restriction of $g$ to $V_{1}$ may have order less than $n$. Indeed, the case $\mathfrak{p}=\mathfrak{g}$ and $n=2$ was considered in [30], and clearly, $g=i d$ on $V_{1}$.

We will apply the theorem above to a holomorphic VOA $U$ of central charge 24 such that

- $U \cong\left(\widetilde{V_{N}}\right)_{g}$ for some Niemeier lattice $N$ and an order $n$ automorphism $g$ of $V_{N}$;

- an order $n$ automorphism of $U$ associated with the $\mathbb{Z}_{n}$-grading of $U$ as a simple current extension of $\left(V_{N}\right)^{g}$ is inner.

We need to confirm the assumptions of Theorem 6.5 for $\mathfrak{g}=U_{1}, \mathfrak{p}=$ $\left(V_{N}\right)_{1}^{g}$, and $W=V_{N}$. Let $V$ be an arbitrary holomorphic VOA of central charge 24 such that $V_{1}=\mathfrak{g}$. Let $u$ be a semisimple element of $\mathfrak{g}$ such that $\mathfrak{g}^{\sigma_{u}}=\mathfrak{p}$. First, by using the representation theory of simple affine VOAs at positive levels (cf. Section 4.2), we try to prove the following:

- The order of $\sigma_{u}$ is $n$ on $V$.

- $\sigma_{u}$ satisfies the conditions (I) and (II) for the orbifold construction.

Next, by using the arguments as in Section 4.3, including the dimension formula (Theorem 4.7), we try to prove that 
- $\widetilde{V}_{\sigma_{u}}$ is isomorphic to $V_{N}$.

Finally, let $\varphi$ be an order $n$ automorphism of $V_{N}$ associated with the simple current extension of $V^{\sigma_{u}}$. We also need to prove the following:

- The conjugacy class $\varphi$ is uniquely determined by the conditions (A) and (B).

Remark 6.7. Note that the automorphism group of $V_{N}$ is determined in [20]. In fact, the automorphism group of a holomorphic VOA of central charge 24 has been determined only for the Niemeier lattice VOA $V_{N}$ and the Moonshine VOA $V^{\natural}$.

Up to now, the uniqueness of the following 17 holomorphic VOAs of $c=24$ has been proved in $[30,37]$ by using the reverse orbifold construction and the argument above.

Theorem 6.8. A strongly regular holomorphic VOA of central charge 24 is unique up to isomorphism if the weight one Lie algebra is one of the 17 types in Proposition 5.4 and Theorem 5.19 except for the trivial Lie algebra 0.

Note that the case $A_{1,2}^{16}$ was already established in Corollary 6.2.

Remark 6.9. The technique of "reverse orbifold" can actually be applied to many more cases, for example, a holomorphic VOA with the weight one Lie algebra $A_{4,5}^{2}$ or $A_{6,7}$. The difficult part is to show that the conjugacy class of the automorphism $\varphi \in \operatorname{Aut}\left(V_{N}\right)$ associated with the simple current extension of $V^{g}$ is uniquely determined by the conditions (A) and (B), which usually requires some non-trivial calculations.

Remark 6.10. After submission of this article, by using the similar techniques as in Theorem 6.5, the uniqueness of holomorphic VOAs of central charge 24 has been proved for 13, 5, 3, and 6 cases in [23], [38], [32], and [39], respectively. Therefore, the uniqueness for 70 of the 71 cases have been established. The remaining task is to prove that a holomorphic VOA of central charge 24 with trivial weight one space is isomorphic to the moonshine VOA, which is a famous conjecture [25].

\section{Acknowledgement.}

The authors wish to thank the referee for helpful comments and suggestions. 


\section{Appendix. Table of 71 holomorphic VOAs of central charge 24}

In this appendix, we give a list of 71 holomorphic VOAs of central charge 24 .

Table 2: 71 holomorphic VOAs of central charge 24

\begin{tabular}{|c|c|c|c|c|c|c|c|}
\hline $\operatorname{dim}\left(V_{1}\right)$ & Lie algebra & rank & Existence & $\operatorname{dim}\left(V_{1}\right)$ & Lie algebra & rank & Existence \\
\hline 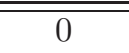 & $\overline{0}$ & $\overline{0}$ & $\overline{[25]}$ & $\overline{24}$ & 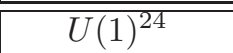 & $\overline{24}$ & $\overline{[25]}$ \\
\hline 36 & $C_{4,10}$ & 4 & {$[22]$} & 36 & $A_{2,6} D_{4,12}$ & 6 & {$[22]$} \\
\hline 36 & $A_{1,4}{ }^{12}$ & 12 & {$[7]$} & 48 & $A_{6,7}$ & 6 & {$[36]$} \\
\hline 48 & $A_{4,5}{ }^{2}$ & 8 & {$[22]$} & 48 & $A_{2,3}{ }^{6}$ & 12 & {$[52]$} \\
\hline 48 & $A_{1,2} D_{5,8}$ & 6 & [31] & 48 & $A_{1,2} A_{5,6} C_{2,3}$ & 8 & [35] \\
\hline 48 & $A_{1,2} A_{3,4}{ }^{3}$ & 10 & [31] & 48 & $A_{1,2}{ }^{16}$ & 16 & {$[7]$} \\
\hline 60 & $C_{2,2}{ }^{6}$ & 12 & {$[7]$} & 60 & $A_{2,2} F_{4,6}$ & 6 & [32] \\
\hline 60 & $A_{2,2}{ }^{4} D_{4,4}$ & 12 & [33] & 72 & $A_{1,1} C_{5,3} G_{2,2}$ & 8 & [22] \\
\hline 72 & $A_{1,1}{ }^{2} D_{6,5}$ & 8 & [35] & 72 & $A_{1,1}{ }^{2} C_{3,2} D_{5,4}$ & 10 & [31] \\
\hline 72 & $A_{1,1}{ }^{3} A_{7,4}$ & 10 & [31] & 72 & $A_{1,1}{ }^{3} A_{5,3} D_{4,3}$ & 12 & [52] \\
\hline 72 & $A_{1,1}{ }^{4} A_{3,2}{ }^{4}$ & 16 & [7] & 72 & $A_{1,1}{ }^{24}$ & 24 & [25] \\
\hline 84 & $B_{3,2}{ }^{4}$ & 12 & [7] & 84 & $A_{4,2}{ }^{2} C_{4,2}$ & 12 & [33] \\
\hline 96 & $C_{2,1}{ }^{4} D_{4,2}{ }^{2}$ & 16 & {$[7]$} & 96 & $A_{2,1} C_{2,1} E_{6,4}$ & 10 & {$[22]$} \\
\hline 96 & $A_{2,1}{ }^{2} A_{8,3}$ & 12 & {$[35]$} & 96 & $A_{2,1}{ }^{2} A_{5,2}{ }^{2} C_{2,1}$ & 16 & {$[31]$} \\
\hline 96 & $A_{2,1}{ }^{12}$ & 24 & {$[25]$} & 108 & $B_{4,2}{ }^{3}$ & 12 & {$[7]$} \\
\hline 120 & $E_{6,3} G_{2,1}{ }^{3}$ & 12 & {$[46]$} & 120 & $A_{3,1} D_{7,3} G_{2,1}$ & 12 & [35] \\
\hline 120 & $A_{3,1} C_{7,2}$ & 10 & {$[31]$} & 120 & $A_{3,1} A_{7,2} C_{3,1}{ }^{2}$ & 16 & [31] \\
\hline 120 & $A_{3,1}{ }^{2} D_{5,2}{ }^{2}$ & 16 & {$[7]$} & 120 & $A_{3,1}{ }^{8}$ & 24 & [25] \\
\hline 132 & $A_{8,2} F_{4,2}$ & 12 & [33] & 144 & $C_{4,1}{ }^{4}$ & 16 & [7] \\
\hline 144 & $B_{3,1}{ }^{2} C_{4,1} D_{6,2}$ & 16 & {$[7]$} & 144 & $A_{4,1} A_{9,2} B_{3,1}$ & 16 & [33] \\
\hline 144 & $A_{4,1}{ }^{6}$ & 24 & {$[25]$} & 156 & $B_{6,2}{ }^{2}$ & 12 & [7] \\
\hline 168 & $D_{4,1}{ }^{6}$ & 24 & [25] & 168 & $A_{5,1} E_{7,3}$ & 12 & [35] \\
\hline 168 & $A_{5,1} C_{5,1} E_{6,2}$ & 16 & {$[33]$} & 168 & $A_{5,1}{ }^{4} D_{4,1}$ & 24 & $25]$ \\
\hline 192 & $B_{4,1} C_{6,1}{ }^{2}$ & 16 & [31] & 192 & $B_{4,1}{ }^{2} D_{8,2}$ & 16 & [7] \\
\hline 192 & $A_{6,1}{ }^{4}$ & 24 & [25] & 216 & $A_{7,1} D_{9,2}$ & 16 & [7] \\
\hline 216 & $A_{7,1}{ }^{2} D_{5,1}{ }^{2}$ & 24 & [25] & 240 & $C_{8,1} F_{4,1}{ }^{2}$ & 16 & [33] \\
\hline 240 & $B_{5,1} E_{7,2} F_{4,1}$ & 16 & [33] & 240 & $A_{8,1}{ }^{3}$ & 24 & [25] \\
\hline 264 & $D_{6,1}{ }^{4}$ & 24 & {$[25]$} & 264 & $A_{9,1}{ }^{2} D_{6,1}$ & 24 & [25] \\
\hline 288 & $B_{6,1} C_{10,1}$ & 16 & {$[33]$} & 300 & $B_{12,2}$ & 12 & [7] \\
\hline 312 & $E_{6,1}{ }^{4}$ & 24 & [25] & 312 & $A_{11,1} D_{7,1} E_{6,1}$ & 24 & {$[25]$} \\
\hline 336 & $A_{12,1}{ }^{2}$ & 24 & [25] & 360 & $D_{8,1}{ }^{3}$ & 24 & [25] \\
\hline 384 & $B_{8,1} E_{8,2}$ & 16 & {$[33]$} & 408 & $A_{15,1} D_{9,1}$ & 24 & {$[25]$} \\
\hline 456 & $D_{10,1} E_{7,1}{ }^{2}$ & 24 & [25] & 456 & $A_{17,1} E_{7,1}$ & 24 & [25] \\
\hline 552 & $D_{12,1^{2}}$ & 24 & [25] & 624 & $A_{24,1}$ & 24 & [25] \\
\hline 744 & $E_{8,1}{ }^{3}$ & 24 & [25] & 744 & $D_{16,1} E_{8,1}$ & 24 & [25] \\
\hline 1128 & $D_{24,1}$ & 24 & [25] & & & & \\
\hline
\end{tabular}




\section{References}

1. K. Betsumiya and A. Munemasa, On triply even binary codes, J. London Math. Soc., 86 (2012), 1-16.

2. R. E. Borcherds, Vertex algebras, Kac-Moody algebras, and the Monster, Proc. Nat'l. Acad. Sci. U.S.A., 83 (1986), 3068-3071.

3. S. Carnahan and M. Miyamoto, Regularity of fixed-point vertex operator subalgebras; arXiv:1603.05645.

4. H. Y. Chen, C.H. Lam and H. Shimakura, $\mathbb{Z}_{3}$-orbifold construction of the Moonshine vertex operator algebra and some maximal 3-local subgroups of the Monster, Math. Z., 288 (2018), 75-100.

5. J. H. Conway, A characterisation of Leech's lattice, Invent. Math., 7 (1969), 137-142.

6. J. H. Conway and N. J. A. Sloane, Sphere Packings, Lattices and Groups, 3rd Edition, Springer, New York, 1999.

7. L. Dolan, P. Goddard and P. Montague, Conformal field theories, representations and lattice constructions, Comm. Math. Phys., 179 (1996), 61-120.

8. C. Dong, Vertex algebras associated with even lattices, J. Algebra, 161 (1993), 245265.

9. C. Dong, R. L. Griess and G. Höhn, Framed vertex operator algebras, codes and Moonshine module, Comm. Math. Phys., 193 (1998), 407-448.

10. C. Dong, X. Jiao and F. Xu, Mirror Extensions of Vertex Operator Algebras, Comm. Math. Phys., 329, (2014), 263-294

11. C. Dong and J. Lepowsky, The algebraic structure of relative twisted vertex operators, J. Pure Appl. Algebra, 110 (1996), 259-295.

12. C. Dong, H. Li and G. Mason, Simple currents and extensions of vertex operator algebras, Comm. Math. Phys., 180 (1996), 671-707.

13. C. Dong, H. Li and G. Mason, Modular-invariance of trace functions in orbifold theory and generalized Moonshine, Comm. Math. Phys., 214 (2000), 1-56.

14. C. Dong and G. Mason, The construction of the moonshine module as a $Z_{p}$-orbifold, in Mathematical aspects of conformal and topological field theories and quantum groups (South Hadley, MA, 1992), Contemp. Math., 175, Amer. Math. Soc., Providence, RI, 1994, 37-52.

15. C. Dong and G. Mason, Holomorphic vertex operator algebras of small central charge, Pacific J. Math., 213 (2004), 253-266.

16. C. Dong and G. Mason, Rational vertex operator algebras and the effective central charge, Int. Math. Res. Not., (2004), 2989-3008.

17. C. Dong and G. Mason, Integrability of $C_{2}$-cofinite vertex operator algebras, Int. Math. Res. Not., (2006), Art. ID 80468, 15 pp. 
18. C. Dong and G. Mason, Shifted vertex operator algebras, Math. Proc. Cambridge Philos. Soc., 141 (2006), 67-80.

19. C. Dong, G. Mason and Y. Zhu, Discrete series of the Virasoro algebra and the moonshine module, Proc. Sympos. Pure Math., 56 (1994), 295-316.

20. C. Dong and K. Nagatomo, Automorphism groups and twisted modules for lattice vertex operator algebras, in Recent developments in quantum affine algebras and related topics (Raleigh, NC, 1998), 117-133, Contemp. Math. 248, Amer. Math. Soc., Providence, RI, 1999.

21. C. Dong, L. Ren and F. Xu, On orbifold theory, Adv. Math., 321 (2017), 1-30.

22. J. van Ekeren, S. Möller and N. Scheithauer, Construction and classification of holomorphic vertex operator algebras, J. Reine Angew. Math. (Published Online).

23. J. van Ekeren, S. Möller and N. Scheithauer, Dimension formulae in genus zero and uniqueness of vertex operator algebras, Internat. Math. Res. Notices (to appear); arXiv:1704.00478.

24. I. B. Frenkel, Y. Huang and J. Lepowsky, On axiomatic approaches to vertex operator algebras and modules, Mem. Amer. Math. Soc., 104 (1993), viii+64 pp.

25. I. Frenkel, J. Lepowsky and A. Meurman, Vertex operator algebras and the Monster, Pure and Appl. Math., Vol.134, Academic Press, Boston, 1988.

26. I. Frenkel and Y. Zhu, Vertex operator algebras associated to representations of affine and Virasoro algebras, Duke Math. J., 66 (1992), 123-168.

27. G. Höhn, Selbstduale Vertexoperatorsuperalgebren und das Babymonster, Dissertation, Bonn, 1995.

28. G. Höhn and N. R. Scheithauer, A generalized Kac-Moody algebra of rank 14, J. Algebra, 404 (2014), 222-239.

29. M. Ishii, D. Sagaki and H. Shimakura, Automorphisms of Niemeier lattices for Miyamoto's $\mathbb{Z}_{3}$-orbifold construction, Math. Z., 280 (2015), 55-83.

30. K. Kawasetsu, C. H. Lam and X. Lin, $\mathbb{Z}_{2}$-orbifold construction associated with $(-1)$ isometry and uniqueness of holomorphic vertex operator algebras of central charge 24, Proc. Amer. Math. Soc. 146 (2018), 1937-1950.

31. C. H. Lam, On the constructions of holomorphic vertex operator algebras of central charge 24, Comm. Math. Phys., 305 (2011), 153-198.

32. C. H. Lam and X. Lin, A Holomorphic vertex operator algebra of central charge 24 with weight one Lie algebra $F_{4,6} A_{2,2} ;$ arXiv:1612.08123.

33. C. H. Lam and H. Shimakura, Quadratic spaces and holomorphic framed vertex operator algebras of central charge 24, Proc. Lond. Math. Soc., 104 (2012), 540-576.

34. C. H. Lam and H. Shimakura, Classification of holomorphic framed vertex operator algebras of central charge 24, Amer. J. Math. 137 (2015), 111-137. 
35. C. H. Lam and H. Shimakura, Orbifold construction of holomorphic vertex operator algebras associated to inner automorphisms, Comm. Math. Phys., 342 (2016), 803841.

36. C. H. Lam and H. Shimakura, A holomorphic vertex operator algebra of central charge 24 whose weight one Lie algebra has type $A_{6,7}$, Lett. Math. Phys., 106 (2016), 15751585 .

37. C. H. Lam and H. Shimakura, Reverse orbifold construction and uniqueness of holomorphic vertex operator algebras; arXiv:1606.08979.

38. C. H. Lam and H. Shimakura, On orbifold constructions associated with the Leech lattice vertex operator algebra; arXiv:1705.01281.

39. C. H. Lam and H. Shimakura, Inertia subgroups and uniqueness of holomorphic vertex operator algebras, in preparation.

40. C. H. Lam and H. Yamauchi, On the structure of framed vertex operator algebras and their pointwise frame stabilizers, Comm. Math. Phys., 277 (2008), 237-285.

41. J. Lepowsky, Calculus of twisted vertex operators, Proc. Natl. Acad. Sci. USA, 82 (1985), 8295-8299.

42. H. Li, Symmetric invariant bilinear forms on vertex operator algebras, J. Pure Appl. Algebra, 96 (1994), 279-297.

43. H. Li, Local systems of twisted vertex operators, vertex operator superalgebras and twisted modules, in Moonshine, the Monster, and related topics, 203-236, Contemp. Math. 193, Amer. Math. Soc., Providence, RI, 1996.

44. M. Miyamoto, Griess algebras and conformal vectors in vertex operator algebras, $J$. Algebra, 179 (1996), 523-548.

45. M. Miyamoto, A new construction of the Moonshine vertex operator algebra over the real number field, Ann. of Math., 159 (2004), 535-596.

46. M. Miyamoto, A $\mathbb{Z}_{3}$-orbifold theory of lattice vertex operator algebra and $\mathbb{Z}_{3}$ orbifold constructions, in Symmetries, integrable systems and representations, 319344, Springer Proc. Math. Stat. 40, Springer, Heidelberg, 2013.

47. M. Miyamoto, $C_{2}$-cofiniteness of cyclic-orbifold models, Comm. Math. Phys., 335 (2015), 1279-1286.

48. M. Miyamoto and K. Tanabe, Uniform product of $A_{g, n}(V)$ for an orbifold model $V$ and $G$-twisted Zhu algebra, J. Algebra 274 (2004), 80-96.

49. S. Möller, A cyclic orbifold theory for holomorphic vertex operator algebras and applications, Dissertation, Darmstadt, 2016; arXiv:1611.09843.

50. P. S. Montague, Orbifold constructions and the classification of self-dual $c=24$ conformal field theories, Nuclear Phys. B, 428 (1994), 233-258.

51. H. V. Niemeier, Definite quadratische Formen der Dimension 24 und Diskriminante 1, J. Number Theory, 5 (1973), 142-178. 
52. D. Sagaki and H. Shimakura, Application of a $\mathbb{Z}_{3}$-orbifold construction to the lattice vertex operator algebras associated to Niemeier lattices, Trans. Amer. Math. Soc., 368 (2016), 1621-1646.

53. A. N. Schellekens, Meromorphic $c=24$ conformal field theories, Comm. Math. Phys., 153 (1993), 159-185.

54. H. Shimakura, The automorphism group of the vertex operator algebra $V_{L}^{+}$for an even lattice $L$ without roots, J. Algebra, 280 (2004), 29-57.

55. B. B. Venkov, On the classification of integral even unimodular 24-dimensional quadratic forms, Algebra, number theory and their applications, Trudy Mat. Inst. Steklov, 148 (1978), 65-76, 273.

56. F. Xu, Mirror extensions of local nets, Comm. Math. Phys., 270 (2007), 835-847.

57. Y. Zhu, Modular invariance of characters of vertex operator algebras, J. Amer. Math. Soc., 9 (1996), 237-302. 